\title{
Efeito da Adição de PHB na Cristalização a Frio do PET
}

\author{
Ana C. Brito, Glória M. Vinhas, Yêda M. B. de Almeida, Renate M. R. Wellen \\ Departamento de Engenharia Química, UFPE \\ Eduardo L. Canedo, Marcelo S. Rabello \\ Unidade Acadêmica de Engenharia de Materiais, UFCG
}

\begin{abstract}
Resumo: O presente trabalho investigou o efeito da adição de baixas concentrações $(0,5$ e 1,0\%) de poli(3-hidroxibutirato) (PHB) na cristalização isotérmica a frio do poli(tereftalato de etileno) (PET). As curvas de energia foram obtidas por calorimetria exploratória diferencial (DSC) e a cinética de cristalização foi analisada pelo método de Avrami. Dois estágios de cristalização foram observados. Os resultados mostraram que o PHB promove alterações na taxa de cristalização do PET, como verificado por mudanças nos tempos para se atingir 1, 10, 30, 50 e 99\% de cristalinidade relativa. As energias de ativação para o primeiro e segundo estágios da cristalização foram determinadas através das curvas de Arrhenius, sendo praticamente independentes da presença de PHB nas misturas PET/PHB. As temperaturas de fusão de equilíbrio foram determinadas de acordo com o procedimento de Hoffman e Weeks; as misturas PET/PHB apresentaram valores mais elevados deste parâmetro sugerindo que o PHB altera a estrutura cristalina do PET.
\end{abstract}

Palavras-chave: Cristalização a frio, PET, PHB, blendas.

\section{Effect of PHB on Cold Crystallization of PET}

Abstract: The present work is concerned with the effect of low concentrations ( 0.5 e $1.0 \%)$ of poly(3-hydroxybutirate) (PHB) on the isothermal cold crystallization of poly(ethylene terephthalate) (PET). Energy curves were obtained by differential scanning calorimetry (DSC) and the kinetics of the crystallization was analyzed using Avrami's method. Two crystallization stages were observed. Results showed that the presence of PHB affects the crystallization rate of PET, as verified by changes in the time needed to reach 1, 10, 30, 50 and $99 \%$ of crystallinity. Activation energies for the two crystallization stages were computed using Arrhenius plots; PET/PHB mixtures showed slightly smaller values. The equilibrium melting temperatures were estimated according to the procedure of Hoffman and Weeks; the higher values obtained for the PET/PHB mixtures suggest that PHB affects the crystalline structure of PET.

Keywords: Cold crystallization, PET, PHB, blends.

\section{Introdução}

Observando não só os benefícios, mas também os danos que algumas tecnologias vêm causando ao meio ambiente e, portanto à própria subsistência do homem moderno, pesquisadores vêm direcionando seus estudos na busca de alternativas que possibilitem o uso dos novos produtos aliados a uma minimização do prejuízo a natureza. Técnicas de reciclagem e reuso de polímeros estão sendo investigadas de modo que se consiga uma diminuição e/ou re-aproveitamento de resinas sintéticas. Outro campo de pesquisa que desponta como grande vigor é a produção e utilização dos polímeros biodegradáveis e/ou obtidos a partir de fontes renováveis.

Atualmente o poli (tereftalato de etileno) (PET) é um poliéster de grande consumo, o qual é muito utilizado em embalagens, principalmente em recipientes descartáveis para indústria alimentícia e em utensílios domésticos. Porém, como esses produtos apresentam um rápido descarte, tem-se observado uma grande quantidade de garrafas e outras embalagens de PET descartadas aleatoriamente, contribuindo para o aumento da poluição dos centros urbanos ${ }^{[1,2]}$.

O PET é um termoplástico de engenharia muito utilizado em diferentes segmentos industriais por apresentar propriedades balanceadas como boa resistência a tração e ao impacto, rigidez, transparência e principalmente baixa permeabilidade a gases, aliadas a um custo relativamente baixo. O poli(3-hidroxibutirato) (PHB) é um poliéster termoplástico biodegradável sintetizado a partir de produtos naturais que apresenta propriedades mecânicas e físicas comparáveis a polímeros produzidos sinteticamente a partir do petróleo, como o polipropileno ${ }^{[3,4]}$.
Como consequência de sua natureza semicristalina, as propriedades finais dos produtos à base de PET são também dependentes de fatores estruturais, tais como: o rearranjo das macromoléculas, o tamanho e perfeição dos cristalitos, tamanho e distribuição das regiões amorfas e cristalinas. Torna-se então muito importante o estudo e análise das condições de processamento ao qual a matéria prima foi submetida como também dos processos de cristalização do PET a fim não apenas de entender, mas também melhorar as propriedades finais dos produtos ${ }^{[4,5]}$.

Sob influência da temperatura o PET pode cristalizar por resfriamento a partir do estado fundido "melt" (cristalização a quente) ou por aquecimento a partir do estado sólido-vítreo (cristalização a frio), como ilustrado na Figura $1^{[6]}$. Na produção de garrafas, por exemplo, utiliza-se um molde gelado para obter pré-formas amorfas. Essas são posteriormente aquecidas em temperatura acima da temperatura de transição vítrea até atingir um estado borrachoso, quando então são submetidas a um processo simultâneo de estiramento e sopro. Durante o aquecimento da préforma a ocorrência de cristalização a frio é altamente indesejável e se constitui na principal causa de refugos de embalagens de PET. Isto ocorre porque nas regiões cristalizadas a pré-forma não adquire maleabilidade suficiente para ser soprada uma vez que o módulo elástico do PET semicristalino é muito superior ao do PET amorfo ${ }^{[7,8]}$. A Figura 2 ilustra algumas pré-formas de PET 
com ocorrência de cristalização a frio (zonas esbranquiçadas) e uma garrafa (com zonas cristalizadas a frio) após ser submetida ao sopro.

Visando o entendimento dos processos de cristalização do PET e buscando alternativas que contribuam para um melhor desempenho de seus produtos, esse trabalho investigou o efeito da adição de PHB em baixas concentrações na cristalização do PET. A cristalização foi estudada por DSC em condições isotérmicas e os parâmetros cinéticos foram determinados utilizando o modelo de Avrami ${ }^{[9]}$. Esse trabalho continua em desenvolvimento onde também está sendo analisado o efeito do PHB na cristalização não isotérmica, nas condições de processamento, propriedades mecânicas e morfologia do PET.

\section{Procedimento Experimental}

\section{Materiais}

O PET usado foi o Cleartuf ${ }^{\circledR}$ fornecido pela $M \& G$, com viscosidade intrínseca de $0,8 \mathrm{dL} \cdot \mathrm{g}^{-1}$. O PHB utilizado foi o FE-113 produzido pela PHB Industrial SA (Brasil) $\operatorname{com} \bar{M}_{w}=340 \mathrm{~kg} \cdot \mathrm{mol}^{-1}$, MFI $=30 \mathrm{dg} / \mathrm{min}\left(2,16 \mathrm{~kg} / 190^{\circ} \mathrm{C}\right)$ de acordo com ASTM D-1238.

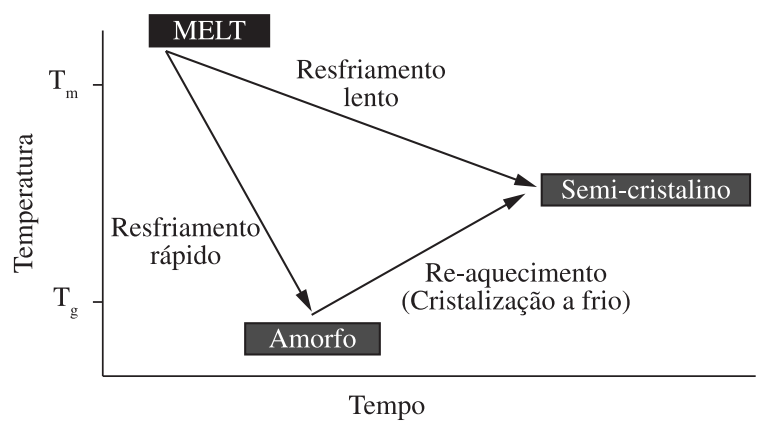

Figura 1. Influência da temperatura e da forma de resfriamento na estrutura cristalina do PET.

\section{Metodologia}

\section{Obtenção das blendas}

Antes do processo de mistura o PHB foi seco em estufa com circulação forçada de ar a $80{ }^{\circ} \mathrm{C}$ por 14 horas, o PET foi seco a $110^{\circ} \mathrm{C}$ durante 6 horas, a fim de retirar a umidade e evitar a hidrólise. As resinas foram misturadas no misturador interno Rheomix 600 (acoplado a um reômetro de torque System-90 da Haake-Büchler), operando com rotores de alta intensidade (tipo roller) e um fator de enchimento de $70 \%$. O PET e o PHB foram misturados a $260{ }^{\circ} \mathrm{C}$ e $60 \mathrm{rpm}$ durante 10 minutos. Ao saírem da câmera de mistura as composições foram imediatamente resfriadas em água/gelo; dessa forma blendas amorfas foram obtidas ${ }^{[10,11]}$.

Foram preparadas composições com 0,5 e 1,0\% de PHB (valores calculados em peso). Uma composição com 100\% de PET também foi submetida às mesmas condições de mistura a fim de comparar os resultados das blendas com a resina PET pura.

\section{Calorimetria exploratória diferencial (DSC)}

No estudo da cristalização isotérmica a frio foram analisadas temperaturas no intervalo entre 110 a $150{ }^{\circ} \mathrm{C}$. Durante o estudo da cristalização as amostras foram submetidas a um aquecimento rápido $\left(\sim 100{ }^{\circ} \mathrm{C} / \mathrm{min}\right)$ da temperatura ambiente até a temperatura de cristalização. A exoterma de cristalização foi obtida em função do tempo até que nenhuma variação com relação a linha base fosse observada. As transições exotérmicas e endotérmicas ocorridas durante o aquecimento das amostras foram obtidas utilizando-se o equipamento Shimadzu DSC-50.

\section{Cristalização isotérmica a frio}

Com as exotermas de cristalização obtidas no DSC foram plotadas as curvas para o desenvolvimento da cristalinidade com o tempo de acordo com a Equação 1:

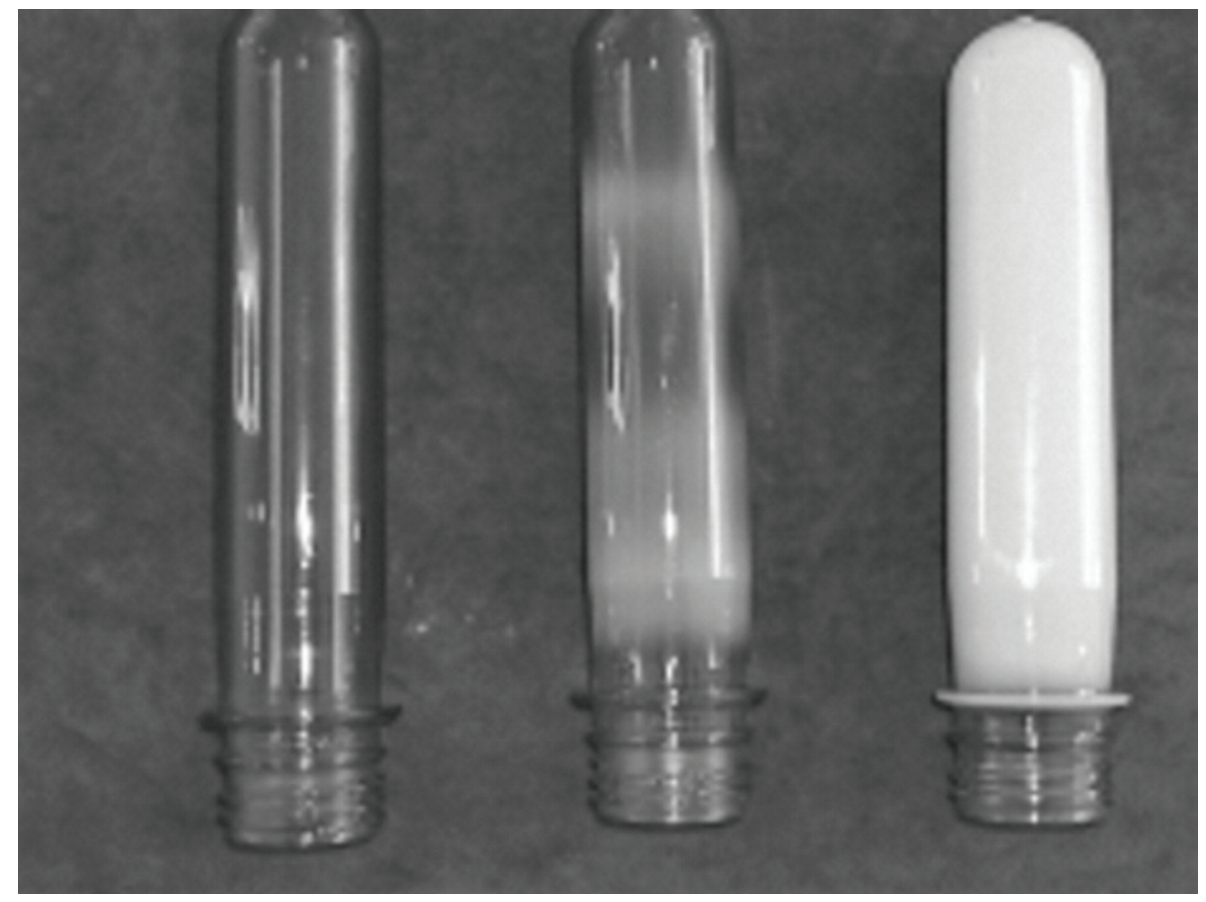

(a)

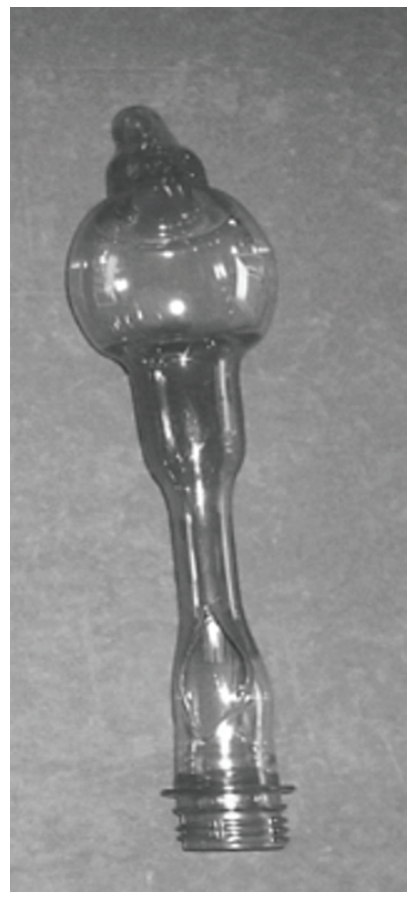

(b)

Figura 2. (a) Pré-formas de PET amorfo, com zonas esbranquiçadas pela cristalização a frio. (b) Garrafa defeituosa após o sopro, devido à presença de regiões cristalizadas na pré-forma. 


$$
x=\frac{\int_{t_{0}}^{t}\left[J\left(t^{\prime}\right)-J_{0}\left(t^{\prime}\right)\right] d t^{\prime}}{\int_{t_{0}}^{t_{\infty}}\left[J\left(t^{\prime}\right)-J_{0}\left(t^{\prime}\right)\right] d t^{\prime}}
$$

onde $x$ é a cristalinidade relativa (ou fracionária), $J(t)$ é a potência liberada medida no DSC, $J_{0}(t)$ é a linha base, $t_{0}$ é tempo onde se inicia a cristalização é to é tempo onde finaliza. O numerador da Equação 1 corresponde a área da exoterma de cristalização acima da linha base (baseline) no intervalo de tempo $t_{0}$ até $t$, e denominador corresponde à área total sob a exorterma de cristalização. No restante do trabalho consideram-se os tempos relativos ao valor inicial, que é tomado como zero $t_{0} \equiv 0$.

\section{Resultados e Discussão}

As curvas do desenvolvimento da cristalinidade com o tempo, $x$, obtidas de acordo com a Equação 1 estão mostradas na
Figura 3. Todas as isotermas de cristalização apresentaram forma sigmoidal que caracteriza um processo de transformação de fases sem descontinuidades. As curvas podem ser deslocadas ao longo do eixo do tempo e sobrepostas, indicando que seguem o mesmo mecanismo de cristalização ${ }^{[12,13]}$.

A partir das curvas da Figura 3 foram calculados os tempos para se atingir 1, 10, 30, 50 e 99\% de cristalinidade relativa (a partir do início da cristalização) para as três composições e em todas as temperaturas testadas; os resultados obtidos estão apresentados na Tabela 1.

Os tempos de cristalização da Tabela 1 são proporcionais ao inverso da taxa média de cristalização entre o início e o nível de cristalinidade correspondente. Observa-se que em temperaturas elevadas a cristalização a frio ocorre de forma ligeiramente mais lenta nas blendas que no PET puro, o que pode estar relacionado com a diminuição da viscosidade e/ou com o aumento do

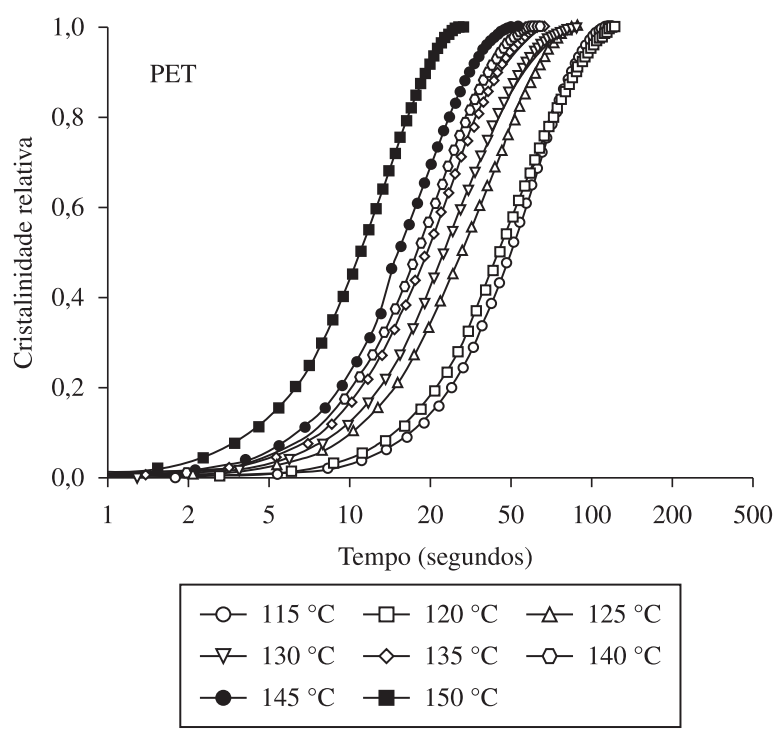

(a)

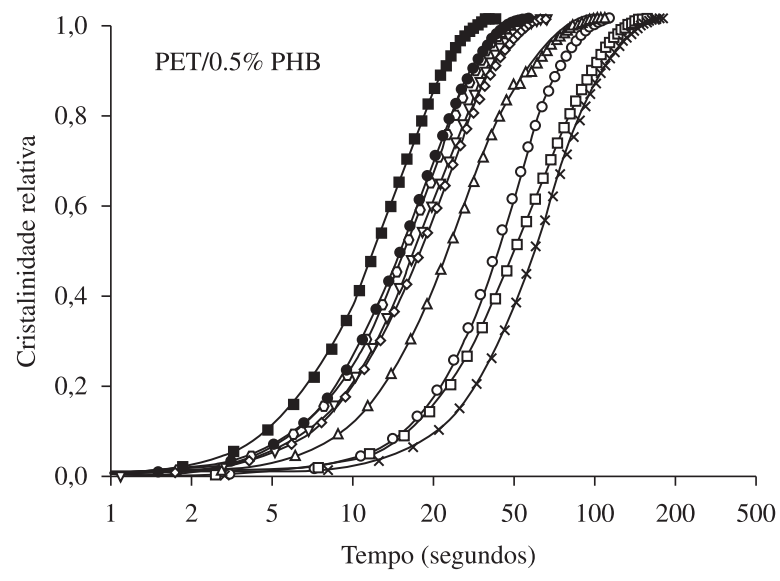

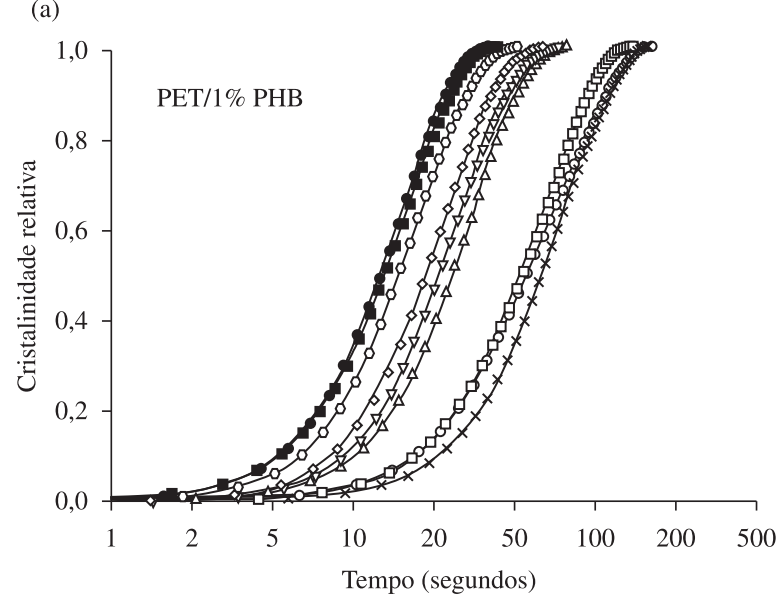

(b)

$$
\begin{aligned}
& \rightarrow 110^{\circ} \mathrm{C} \rightarrow 115^{\circ} \mathrm{C} \rightarrow 120^{\circ} \mathrm{C} \\
& -125^{\circ} \mathrm{C} \rightarrow 130{ }^{\circ} \mathrm{C} \multimap 135^{\circ} \mathrm{C} \\
& \multimap-140{ }^{\circ} \mathrm{C} \rightarrow 145^{\circ} \mathrm{C} \rightarrow 150^{\circ} \mathrm{C}
\end{aligned}
$$

(c)

Figura 3. Desenvolvimento da cristalinidade relativa com o tempo de cristalização das composições PET (a), PET/0,5\% PHB (b), e PET/1\% PHB (c). As temperaturas de cristalização estão indicadas. 
Tabela 1. Tempos necessários para se atingir diferentes valores da cristalinidade relativa.

$X 110^{\circ} \mathrm{C} 115^{\circ} \mathrm{C} 120^{\circ} \mathrm{C} 125^{\circ} \mathrm{C} 130^{\circ} \mathrm{C} 135^{\circ} \mathrm{C} 140{ }^{\circ} \mathrm{C} 145^{\circ} \mathrm{C} 150{ }^{\circ} \mathrm{C}$ (\%)

\begin{tabular}{lrrrrrrrrr}
\hline \multicolumn{10}{c}{ PET } \\
\hline 1 & - & 5,6 & 4,1 & 2,9 & 2,2 & 1,9 & 2,1 & 1,5 & 1,0 \\
10 & - & 19,0 & 16,3 & 10,6 & 9,1 & 7,9 & 7,1 & 6,1 & 4,0 \\
30 & - & 34,7 & 30,6 & 20,6 & 17,7 & 15,0 & 13,7 & 11,8 & 8,0 \\
50 & - & 48,4 & 43,8 & 30,3 & 25,6 & 21,6 & 19,8 & 17,2 & 11,5 \\
99 & - & 116,2 & 123,3 & 81,9 & 77,2 & 58,5 & 53,5 & 45,1 & 27,6 \\
\hline \multicolumn{7}{c}{ PET/0,5\% PHB } \\
\hline 1 & 6,3 & 5,8 & 5,0 & 2,9 & 1,9 & 2,0 & 1,9 & 1,7 & 1,3 \\
10 & 22,7 & 16,3 & 17,4 & 9,3 & 6,7 & 6,8 & 6,0 & 5,7 & 4,5 \\
30 & 41,1 & 29,0 & 32,8 & 13,7 & 12,8 & 13,2 & 11,5 & 11,1 & 8,7 \\
50 & 57,3 & 39,8 & 47,6 & 25,4 & 18,7 & 19,5 & 16,7 & 16,1 & 12,6 \\
99 & 161,1 & 101,4 & 142,0 & 91,3 & 54,4 & 55,0 & 45,8 & 45,7 & 33,6 \\
\hline \multicolumn{7}{c}{ PET/1\% PHB } \\
\hline 1 & 6,5 & 6,1 & 5,3 & 3,1 & 3,0 & 2,5 & 2,0 & 1,7 & 1,3 \\
10 & 23,1 & 18,2 & 18,3 & 10,3 & 9,4 & 8,1 & 6,1 & 5,0 & 5,0 \\
30 & 43,4 & 35,7 & 35,0 & 18,7 & 16,6 & 14,7 & 11,3 & 9,3 & 9,7 \\
50 & 61,7 & 53,7 & 51,5 & 26,2 & 23,2 & 20,3 & 15,8 & 13,3 & 13,7 \\
99 & 153,2 & 157,9 & 127,2 & 66,8 & 62,1 & 53,3 & 41,5 & 32,7 & 35,1 \\
\hline
\end{tabular}

movimento segmental em temperaturas mais altas. Nas isotermas de cristalização mostradas na Figura 3 e nos dados da Tabela 1, verifica-se alterações nos tempos para se atingir diferentes níveis de cristalinidade, o que pode estar relacionado com a presença de cadeias de baixa massa molar de PHB, atuando como impurezas e modificando o processo de cristalização a frio do PET. Estas impurezas podem ter sido originadas durante a polimerização e/ou como resultado de processos degradativos ocorridos durante a etapa de mistura ${ }^{[13]}$.

Neste trabalho a mistura ocorreu a $260{ }^{\circ} \mathrm{C}$ e de acordo com informações da literatura é sugerido que a degradação do PHB inicia em temperaturas ligeiramente superiores a $180{ }^{\circ} \mathrm{C}$. Portanto, durante a mistura pode ter ocorrido cisão das macromoléculas de PHB e assim moléculas de baixo peso molecular (que podem estar atuando como impurezas) podem ter sido originadas, e estas podem estar perturbando a cristalização a frio do PET ${ }^{[14,15]}$.

\section{Cinética da cristalização isotérmica a frio}

Durante o estudo da cinética de cristalização isotérmica a equação de Avrami ${ }^{[9]}$ foi utilizada na determinação dos parâmetros cinéticos, relacionando a fração de cristalinidade, $x$, com o tempo de cristalização, $t$, de acordo com a Equação 2:

$$
1-x=\exp \left(-K t^{n}\right)
$$

onde $K$ and $n$ são os parâmetros de Avrami. Aplicando-se o duplo logaritmo na Equação 2 obtém-se a Equação 3:

$$
\log [-\ln (1-x)]=\log K+n \log t
$$

O método de Avrami é válido para uma relação linear entre $\log [-\ln (1-x)]$ e $\log (t)$.

A Figura 4 ilustra, como exemplo, uma curva de Avrami para o PET cristalizado a frio a $135^{\circ} \mathrm{C}$. Pode-se observar que a cristalização a frio ocorre em dois estágios, ambos seguindo (aproximadamente) a teoria de Avrami, um processo inicial e um processo final com diferentes parâmetros cinéticos. A ocorrência de comportamento similar foi previamente verificado em trabalhos anteriores de alguns dos atuais autores ${ }^{[7,13,15]}$.

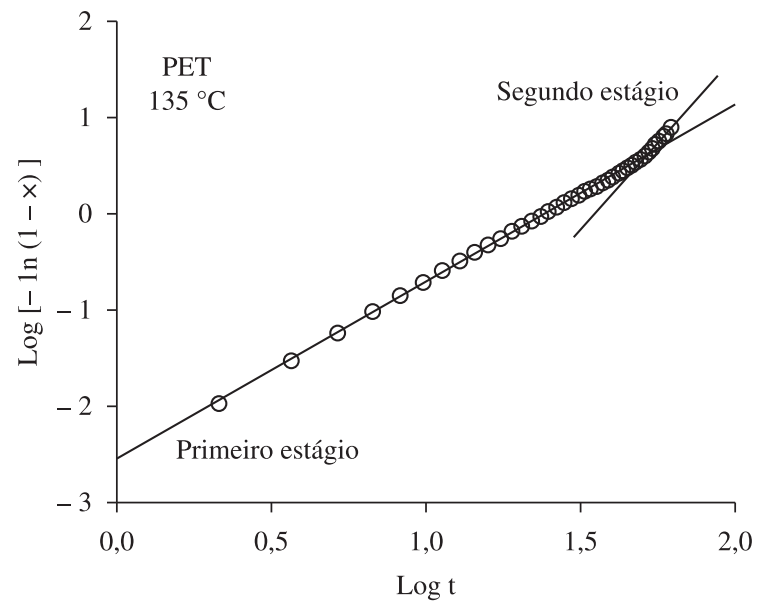

Figura 4. Curva de Avrami para cristalização a frio do PET a $135^{\circ} \mathrm{C}$.

A cristalização em dois estágios foi observada tanto no PET puro como nas blendas com PHB nas diferentes temperaturas de cristalização investigadas; a equação de Avrami foi utilizada para determinar os parâmetros cinéticos nos dois estágios, e os valores obtidos estão apresentados nas Tabelas 2 e 3 . Vale ressaltar que o parâmetro $K$ de Avrami foi expresso nas referidas tabelas em unidades de $\mathrm{s}^{-\mathrm{n}}$ (onde $n$ é o expoente de Avrami).

A constante de Avrami $K$, que está relacionada com o processo de cristalização global, aumenta com a temperatura, tanto no primeiro estágio $\left(K_{1}\right)$ quanto no segundo estágio $\left(K_{2}\right)$, sendo ligeiramente superior para PET puro quando comparado com os valores para as blendas PET/PHB.

O expoente de Avrami $n$, que é influenciado pelas características gerais do processo de cristalização, apresentou, no primeiro estágio $\left(n_{1}\right)$, valores próximos a 2 tanto para o PET como para as blendas com PHB, consistente com nucleação heterogênea, crescimento cristalino bidimensional (discos) controlado pela difusão das macromoléculas ${ }^{[6]}$. Já no segundo estágio $\left(n_{2}\right)$ o expoente de Avrami variou com a temperatura de cristalização, diminuindo de valores próximos a 4 para valores próximos a 2 no intervalo entre $100 \mathrm{e} 150^{\circ} \mathrm{C}$, respectivamente. $\mathrm{O}$ segundo estágio da cristalização ou cristalização secundária ocorre em regiões de menor cristalizabilidade, entre os esferulitos ou dentro dos esferulitos nas regiões desordenadas, estruturas essas formadas durante a cristalização primária. Uma hipótese para o comportamento observado com o expoente de Avrami durante esse estágio de cristalização pode estar relacionada com o fato de que em temperaturas mais elevadas o movimento das macromoléculas é maior, resultando também em maior velocidade de cristalização, mesmo se tratando de níveis elevados de cristalinidade relativa (ver Tabela 1). Esses mecanismos contribuem para formação de cristalitos de menor perfeição e então em valores mais baixos do expoente de Avrami, enquanto que o contrário pode ser verificado em temperaturas de cristalização mais elevadas ${ }^{[6,9,15]}$.

O parâmetro cinético combinado:

$$
k=K^{1 / n}
$$

é utilizado na literatura como uma forma de expressar (aproximadamente) tanto as características cinéticas gerais quanto as específicas no processo de cristalização descrito pela equação de Avrami ${ }^{[16]}$. A Figura 5 mostra a "constante" $k$ como função da temperatura para as amostras de PET puro e da blenda PET/1\% PHB.

Observa-se que, levando em consideração a dispersão dos resultados devido ao erro experimental, o primeiro e segundo 
estágios coincidem numa única reta, neste caso as composições apresentam velocidades ou constantes de cristalização similares no intervalo de temperatura de cristalização isotérmica investigado. A constante cinética $k$ difere em menos que $1 \%$ entre o PET puro e as blendas PET/PHB.

\section{Determinação da energia de ativação da cristalização isotérmica a frio}

É lícito assumir que o processo de cristalização a frio do PET é termicamente ativado ${ }^{[16]}$. Desta forma, a constante cinética $k$

Tabela 2. Parâmetros de Avrami para o primeiro estágio da cristalização do PET e das blendas PET/PHB.

\begin{tabular}{|c|c|c|c|c|c|c|}
\hline \multirow{2}{*}{$\begin{array}{c}T \\
\left({ }^{\circ} \mathbf{C}\right)\end{array}$} & \multicolumn{2}{|c|}{ PET } & \multicolumn{2}{|c|}{ PET/0,5\% PHB } & \multicolumn{2}{|c|}{ PET/1\% PHB } \\
\hline & $\log K 1$ & $n 1$ & $\log K 1$ & $n \mathbf{1}$ & $\log K 1$ & $N 1$ \\
\hline 110 & - & - & $-3,606$ & 1,95 & $-3,591$ & 1,92 \\
\hline 115 & $-3,534$ & 2,00 & $-3,603$ & 2,15 & $-3,230$ & 1,73 \\
\hline 120 & $-3,189$ & 1,84 & $-3,505$ & 2,02 & $-3,424$ & 1,93 \\
\hline 125 & $-2,897$ & 1,86 & $-2,786$ & 1,86 & $-3,089$ & 2,07 \\
\hline 130 & $-2,681$ & 1,78 & $-2,959$ & 1,91 & $-3,043$ & 2,10 \\
\hline 135 & $-2,538$ & 1,77 & $-3,477$ & 1,84 & $-2,970$ & 2,17 \\
\hline 140 & $-2,568$ & 1,86 & $-3,518$ & 1,85 & $-2,564$ & 2,01 \\
\hline 145 & $-2,410$ & 1,80 & $-3,782$ & 1,87 & $-2,354$ & 1,96 \\
\hline 150 & $-2,033$ & 1,77 & $-4,348$ & 2,02 & $-2,301$ & 1,89 \\
\hline
\end{tabular}

Tabela 3. Parâmetros de Avrami para o segundo estágio da cristalização do PET e das blendas PET/PHB.

\begin{tabular}{|c|c|c|c|c|c|c|}
\hline \multirow{2}{*}{$\begin{array}{c}T \\
\left({ }^{\circ} \mathbf{C}\right)\end{array}$} & \multicolumn{2}{|c|}{ PET } & \multicolumn{2}{|c|}{ PET/0,5\% PHB } & \multicolumn{2}{|c|}{ PET/1\% PHB } \\
\hline & $\log K 2$ & $n 2$ & $\log K 2$ & $n 2$ & $\log K 2$ & $N 2$ \\
\hline 110 & - & - & $-4,479$ & 2,33 & $-7,543$ & 3,77 \\
\hline 115 & $-8,055$ & 4,23 & $-3,593$ & 2,12 & $-7,945$ & 3,92 \\
\hline 120 & $-5,456$ & 2,93 & $-3,849$ & 2,10 & $-5,981$ & 3,17 \\
\hline 125 & $-4,865$ & 2,89 & $-2,742$ & 1,75 & $-4,471$ & 2,82 \\
\hline 130 & $-2,910$ & 1,89 & $-2,959$ & 2,09 & $-4,175$ & 2,71 \\
\hline 135 & $-3,639$ & 2,44 & $-3,477$ & 2,39 & $-3,496$ & 2,42 \\
\hline 140 & $-4,587$ & 3,05 & $-3,518$ & 2,52 & $-4,373$ & 3,12 \\
\hline 145 & $-2,468$ & 1,88 & $-3,782$ & 2,69 & $-3,450$ & 2,72 \\
\hline 150 & $-3,226$ & 2,70 & $-4,349$ & 3,30 & $-2,878$ & 2,29 \\
\hline
\end{tabular}

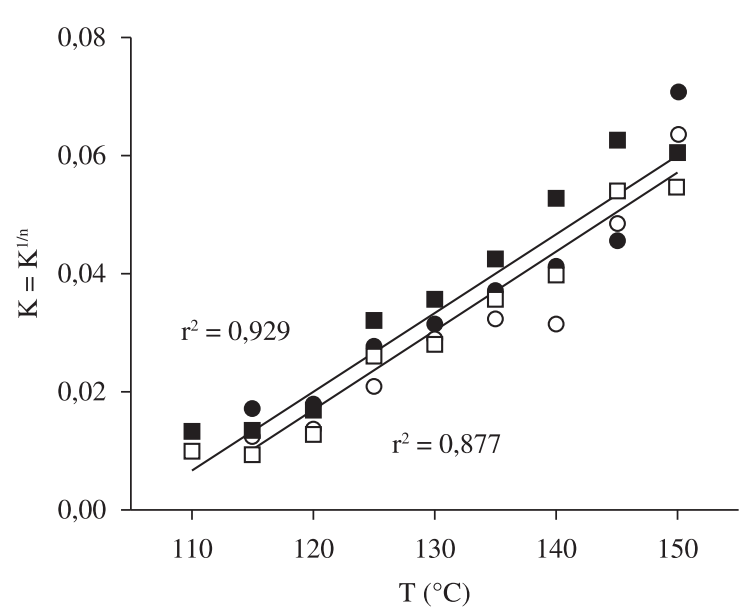

- PET — PET/1\% PHB

Figura 5. Constante cinética para cristalização a frio do PET e da blenda PET/1\%PHB a diferentes temperaturas; símbolos cheios: primeiro estágio; símbolos vazios: segundo estágio; unidades de $k: \mathrm{s}^{-1}$. pode ser aproximadamente descrita de acordo com a Equação 5 de Arrhenius:

$$
k^{1 / n}=k_{0} \exp \left(-\frac{\Delta E}{R T_{c}}\right)
$$

onde $k_{0}$ é um fator pré-exponencial independente da temperatura, $T_{\mathrm{c}}$ é a temperatura (absoluta) de cristalização a frio, $R=8,315 \mathrm{~J} \cdot \mathrm{mol}^{-1} \mathrm{~K}$ é a constante universal dos gases, e $\Delta E$ é a energia de ativação para a cristalização isotérmica. A energia de ativação da cristalização isotérmica pode ser determinada através do gráfico $\ln k$ versus $1 / T_{c}$, onde $\Delta E$ corresponde à inclinação da reta, como mostrado na Figura 6.

As energias de ativação obtidas com este procedimento são praticamente independentes da presença de PHB (53,6 $\pm 2,7 \mathrm{~kJ} \cdot \mathrm{mol}^{-1}$ no primeiro estágio, $56,7 \pm 6,5 \mathrm{~kJ} \cdot \mathrm{mol}^{-1}$ no segundo estágio da cristalização), observando-se que a adição de PHB não alterou o valor energético da cristalização a frio do PET. Valor similar ao $\Delta E$ determinado neste trabalho foi previamente obtido pelos autores ${ }^{[13,17]}$.

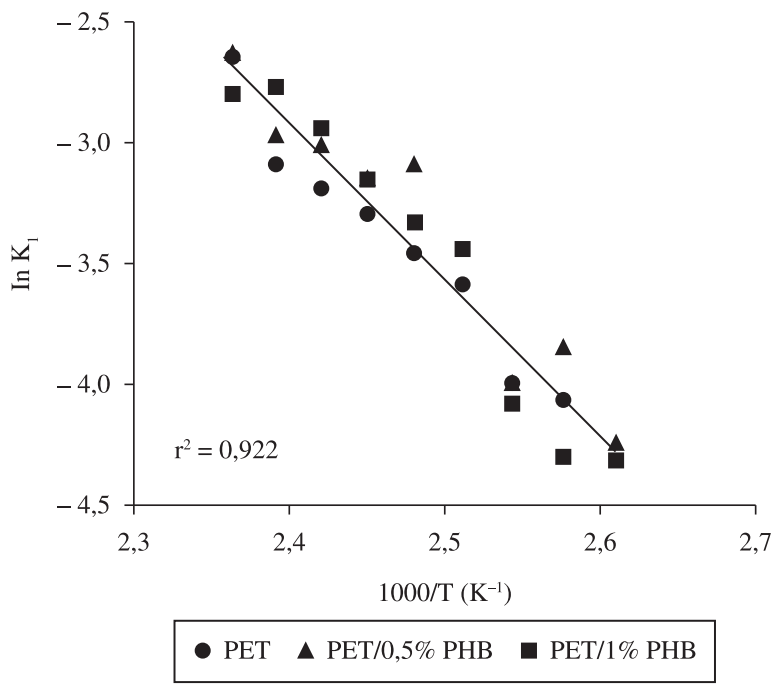

(a)

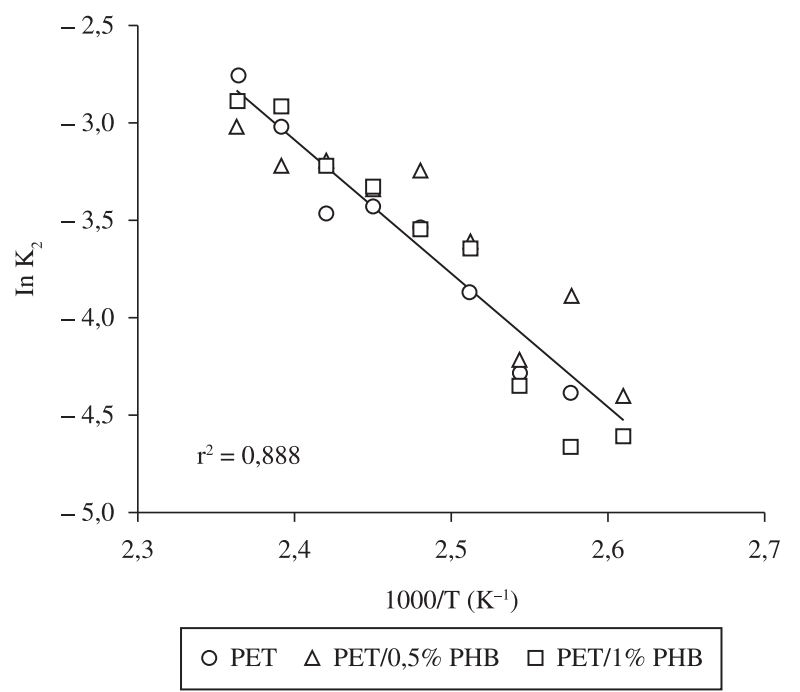

(b)

Figure 6. Representação de Arrhenius utilizada para estimar as energias de ativação da cristalização isotérmica a frio do PET e das blendas PET/PHB; primeiro estágio (a), segundo estágio (b). 

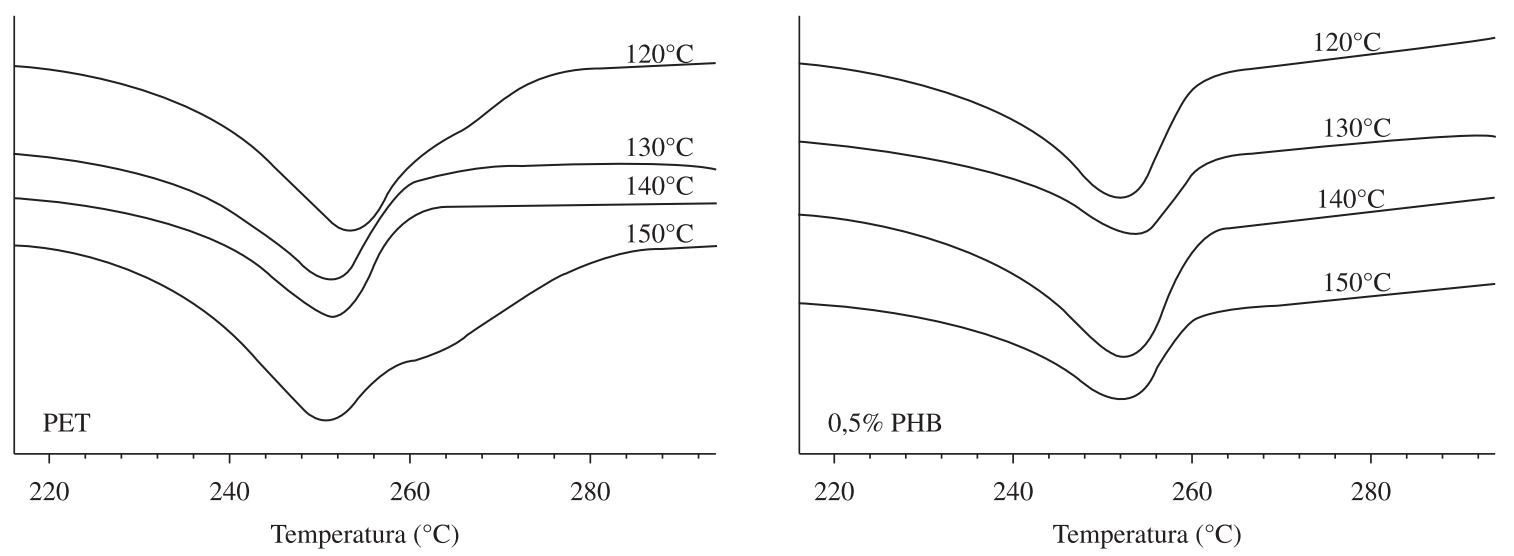

Figura 7. Endotermas de fusão do PET (a) e da blenda com 0,5\% PHB (b), determinadas em amostras previamente cristalizadas isotermicamente a frio nas temperaturas indicadas.

Tabela 4. Temperatura de fusão de equilíbrio, entalpia de fusão e grau de cristalinidade do PET e das blendas PET/PHB. Amostras previamente cristalizadas isotermicamente a frio.

\begin{tabular}{lccc}
\hline Composição & $T_{m}^{o}\left({ }^{\circ} \mathbf{C}\right)$ & $\Delta H_{m}\left(\mathbf{J J}^{-\mathbf{1}}\right)$ & $X_{\infty}(\boldsymbol{\%})$ \\
\hline PET & 251,0 & 35,4 & 30,1 \\
PET/0,5\% PHB & 255,0 & 30,4 & 25,8 \\
PET/1\% PHB & 252,5 & 37,4 & 31,8 \\
\hline
\end{tabular}

\section{Determinação da temperatura de fusão de equilíbrio}

A temperatura de fusão de equilíbrio do PET e das blendas PET/ PHB foi determinada de acordo com o procedimento desenvolvido por Hoffman e Weeks ${ }^{[18]}$. De acordo com esse método a temperatura de fusão de equilíbrio $T_{m}^{0}$ pode ser obtida através do gráfico de $T m$ versus $T c$; a temperatura $T_{m}^{0}$ corresponde a intersecção da curva com a linha $T_{m}=T_{c}$.

A Figura 7 apresenta as endotermas de fusão do PET e da blenda com 0,5\% PHB. A partir dessas endotermas foram obtidas a temperatura de fusão $T_{\mathrm{m}}$ (altura máxima do pico endotérmico) e a entalpia de fusão por unidade de massa $\Delta H_{m}$. O grau de cristalinidade $X_{\infty}$ foi determinado de acordo com a Equação 6. Os resultados obtidos para estes parâmetros estão resumidos na Tabela 4. Pode-se observar que a adição de PHB não promoveu grandes alterações na $T m, \Delta H_{m}$ e $X \infty$ do PET e valores similares foram verificados na resina pura e nas blendas.

$$
X_{\infty}=\frac{\Delta H_{m}}{\Delta H_{m}^{0}}
$$

Onde $\Delta H_{m}^{0}$ é a entalpia de fusão da amostra completamente cristalina; para o PET foi utilizado o valor $\Delta H_{m}^{0}=117,65 \mathrm{~J} \cdot \mathrm{g}^{-1[19]}$.

\section{Conclusões}

Esse trabalho investigou o efeito da adição de baixas concentrações de PHB no comportamento térmico e na cristalização a frio do PET. Os resultados obtidos indicam que a adição de PHB promove mínimas alterações na cristalização a frio do PET. O efeito pode ser atribuído à presença de impurezas ou moléculas de baixo peso molecular originadas por processos degradativos durante a etapa de mistura.

Trabalhos futuros estão sendo realizados empregandose técnicas de análise termogravimétrica, espectroscopia de infravermelho e microscopia ótica, o que pode esclarecer melhor as questões aqui levantadas.

\section{Agradecimentos}

Os autores agradecem a M\&G pelo fornecimento do PET, a PHB Brasil pelo PHB fornecido e a FACEPE pelo apoio financeiro.

\section{Referências Bibliográficas}

1. Piva, A. M. \& Wiebeck, H. - "Reciclagem do Plástico", ArtLiber, São Paulo (2004).

2. Zanin, M. \& Mancini, S.D. -"Resíduos Plásticos e Reciclagem: Aspectos Gerais e Tecnologia", EdUFSCar, São Carlos (2004).

3. Falcone, D. M. B.; Agnelli, J. A. M. \& Faria, L. I. L. - Polímeros, 17, p.5 (2007).

4. Mancini, S. D.; Bezerra, M. N. \& Zanin, M. - Polímeros, 8, p.68 (1998).

5. Di Souza, L.; Torres, M. C. M. \& Ruvolo-Filho, A. - Polímeros, 18, p.334 (2008).

6. Mandelkern, L. - "Crystallization of Polymers. 2: Kinetics and Mechanisms", Cambridge University Press, Cambridge (2004). http:// dx.doi.org/10.1017/CBO9780511535413

7. Wellen, R. M. R. \& Rabello, M. S. - Polímeros, 17, p.113 (2007).

8. Fann, D. M.; Huang, S. K. \& Lee, J. Y. - Polym. Eng. Sci., 38, p.265 (1998). http://dx.doi.org/10.1002/pen.10187

9. Avrami, M. - J. Chem. Phys., 7, p.1103 (1939).

10. Ballara, A. \& Verdu, J. - Polym. Degrad. Stab., 26, p.361 (1989). http:// dx.doi.org/10.1016/0141-3910(89)90114-6

11. Apiwanthanakorn, N.; Supaphol, P. \& Nithitanakul, M. Polym. Test., 23, p.817 (2004). http://dx.doi.org/10.1016/j. polymertesting.2004.03.001

12. Wellen, R. M. R. \& Rabello, M. S. - J. Mater. Sci., 40, p.6099 (2005). http://dx.doi.org/10.1007/s10853-005-3173-3

13. Wellen, R. M. R.- "Cristalização a frio do PET e das blendas PET/PS e PET/SAN", Tese de Doutorado, Universidade Federal de Campina Grande, Brasil (2007).

14. Karlsson, S. \& Albertson, A. C. - Polym. Eng. Sci., 38, p.1251 (1998). http://dx.doi.org/10.1002/pen.10294

15. Wellen, R. M. R. \& Rabello, M. S. - J. Appl. Polym. Sci., 116, p.1077 (2010).

16. Cebe, P. - Polym. Comp., 9, p.271 (1988). http://dx.doi.org/10.1002/ pc.750090405

17. Wellen, R. M. R. \& Rabello, M. S. - J. Appl. Polym. Sci., 114, p.1884 (2009). http://dx.doi.org/10.1002/app.29569

18. Hoffman, J. D. \& Weeks, J. J. - J. Research Natl. Bur. Standards, 66-A, p.13 (1962)

19. Roberts, R. C. - Polym., 10, p.117 (1969). http://dx.doi. org/10.1016/0032-3861(69)90015-9

Enviado: $31 / 01 / 11$

Reenviado: 03/05/11

Aceito: 07/06/11 